\title{
CUTANEOUS TUBERCULOSIS- A RARE OCCURRENCE IN A THREE YEAR OLD
}

\author{
Sowmya Naga Dogiparthi', Vijay Kumar², Jeyakumari Jeevan ${ }^{3}$, G. Srinivasan ${ }^{4}$
}

${ }^{1}$ Assistant Professor, Department of Dermatology, Venereology and Leprosy, Shree Sathya Sai Medical College and Research Institute, Tiruporur, Chennai.

${ }^{2}$ Senior Resident, Department of Dermatology, Venereology and Leprosy, Shree Sathya Sai Medical College and Research Institute, Tiruporur, Chennai.

${ }^{3}$ Professor, Department of Dermatology, Venereology and Leprosy, Shree Sathya Sai Medical College and Research Institute, Tiruporur, Chennai.

${ }^{4}$ HOD, Department of Dermatology, Venereology and Leprosy, Shree Sathya Sai Medical College and Research Institute, Tiruporur, Chennai.

HOW TO CITE THIS ARTICLE: Dogiparthi SN, Kumar V, Jeevan J, et al. Cutaneous tuberculosis- a rare occurrence in a three year old. J. Evolution Med. Dent. Sci. 2017;6(88):6161-6164, DOI: 10.14260/jemds/2017/1338

\section{PRESENTATION OF CASE}

A three-year-old accompanied by his parents came to the Dermatology OPD with presenting complaints of an asymptomatic elevated lesion progressing in size over the medial aspect of right foot adjoining the sole for duration of one year. No history of trauma to the foot. No history of pain or discharge from the lesion. No history of preceding systemic symptoms prior to the onset of lesion. No history of systemic illness or previous exposure to Tuberculosis. No history of Tuberculosis in the family. Parents give history of child being bare foot while playing outside. Immunisation history is up-to-date. No recent history of upper respiratory tract infection or treatment for the cutaneous lesions. On examination of the patient with the parent's consent patient was conscious, oriented and afebrile. No pallor, icterus, lymphadenopathy or oedema. On local examination of the skin, a single hyperkeratotic irregular shaped scaly plaque measuring $5 \times 3 \mathrm{~cm}$ was present over the right foot adjoining the sole. Lesion shows healing at one end and progression over other areas. No other skin lesions elsewhere.

\section{Before Treatment}

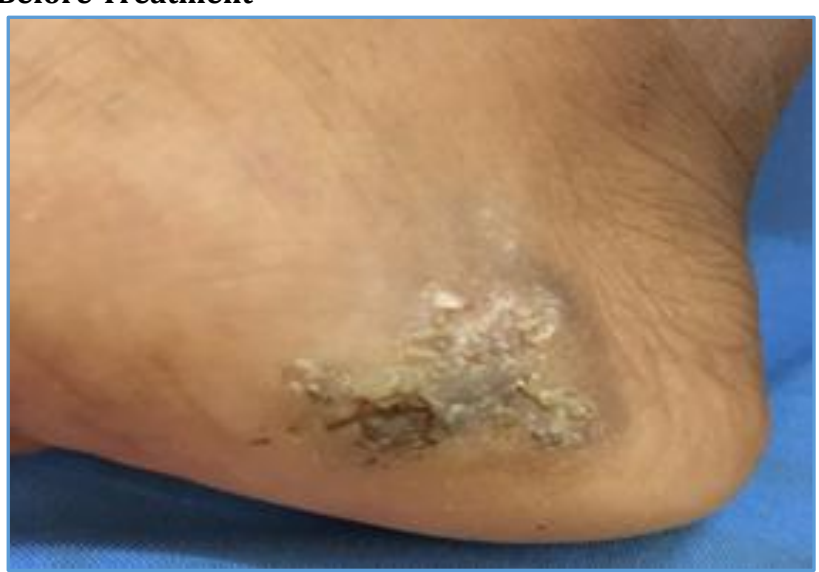

Figure A. 5 x 3 cm Hyperkeratotic Irregular Shaped Scaly Plaque

'Financial or Other Competing Interest': None.

Submission 30-09-2017, Peer Review 24-10-2017,

Acceptance 30-10-2017, Published 06-11-2017.

Corresponding Author:

Dr. Sowmya Naga Dogiparthi,

Dermipure Dermaclinic,

Sai Kuteeram, No. 16, $9^{\text {th }}$ Lane,

Sasthri Nagar, Adayar,

Chennai-600020.

E-mail: sowmy88688@gmail.com

DOI: $10.14260 /$ jemds/2017/1338

\section{(c) (i) $(9)$}

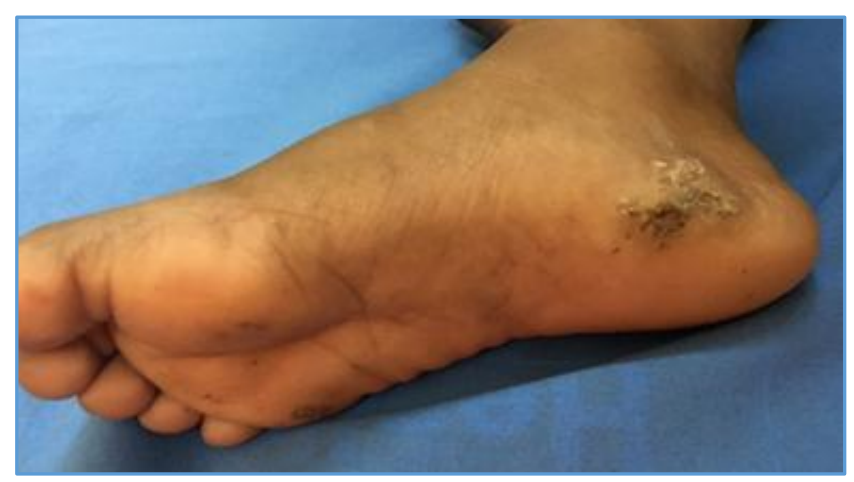

Figure B. Full Image of Right Foot

\section{DIFFERENTIAL DIAGNOSIS}

The diagnosis of cutaneous TB is based on the characteristic clinical morphology of the lesions as well as laboratory tests. Differential diagnosis of lupus vulgaris, mosaic wart, tuberculosis verrucosa cutis and hyperkeratotic eczema was postulated for our case. Common clinical differential diagnoses of skin TB include cutaneous leishmaniasis, leprosy, atypical mycobacterial infections, fungal infections like chromomycosis and sporotrichosis and sarcoidosis. Many of these conditions also show granulomas on histology and thus definitive diagnosis relies on the direct demonstration of tubercle AFB on stained smears or biopsies, isolation by culture or detection by PCR and related molecular techniques.

\section{CLINICAL DIAGNOSIS}

Investigations such as complete blood count (CBC), liver function test, erythrocyte sedimentation rate (ESR), Mantoux test, x-ray of chest, ultrasound of the abdomen and biopsy of the lesion were done. ESR and Mantoux were elevated. The Mantoux test was performed using 1 tuberculin unit $(0.02 \mathrm{mg}$ of purified protein derivative) and read at 72 hours. Induration of more than $10 \mathrm{~mm}$ was suggestive of infection with mycobacterium tuberculosis.

The Mantoux also known as tuberculin test is a screening test to detect the presence or absence of tubercular infection. It is performed by injecting $0.1 \mathrm{~mL}(5 \mathrm{TU})$ of the purified protein derivative (PPD) on the volar surface of the forearm using a 27-gauge needle and measuring the induration after $48-72 \mathrm{~h}$. Induration of $10 \mathrm{~mm}$ or more is considered significant, but indicates infection and not necessarily the disease. False-positive results may occur due to exposure to environmental mycobacteria as well as within 1 year of BCG 
vaccination. False-negative reactions may also be seen in patients with disseminated infection like miliary TB or in immunosuppressed states like severe malnutrition in children or HIV infection. Pandhi et al. reported 66 of 68 children (97.1\%) with positive Mantoux test with induration ranging from 10 to $32 \mathrm{~mm} \cdot{ }^{[1]}$ Kumar et al observed that of their children with cutaneous TB, 91.8\% patients with localised disease had positive Mantoux test as against only $50 \%$ of the cases with disseminated disease.[2] Severe reactions resulting in vesiculation or ulceration have also been observed in children. ${ }^{[1],[3]}$ Tuberculin test is more commonly positive in tuberculids, as reported in all cases of LS by Singal et al. ${ }^{[4]}$ The role of BCG as a preventive measure against cutaneous TB is difficult to define. Protective efficacy of BCG vaccine in TB in various clinical trials has ranged from $0 \%$ to $80 \%{ }^{[5]}$ In a meta-analysis, a high rate of $65 \%-86 \%$ protection against tubercular meningitis and miliary disease was reported.[6] Zodpey et al demonstrated BCG vaccination to be moderately effective in adults against skin TB with an efficacy of $60.9 \% .^{[5]}$ Kumar et al reported that BCG-vaccinated children did not develop disseminated disease. [2] However, others found no significant differences between the vaccinated and the unvaccinated groups.[7],[8] Protective efficacy of BCG has been reported to decrease with time interval since vaccination.

\section{PATHOLOGICAL DISCUSSION}

Skin biopsy was done from the active border of the lesion, which showed markedly hyperkeratotic, hyperplastic and acanthotic squamous epithelium. The upper dermis was diffusely infiltrated by lymphocytes and plasmocytes amidst, which are seen in a few scattered granulomas formed of epithelial cells. Some of the granulomas contained Langhans giant cells and foreign body giant cells. The features were consistent with Tuberculosis verrucosa cutis.

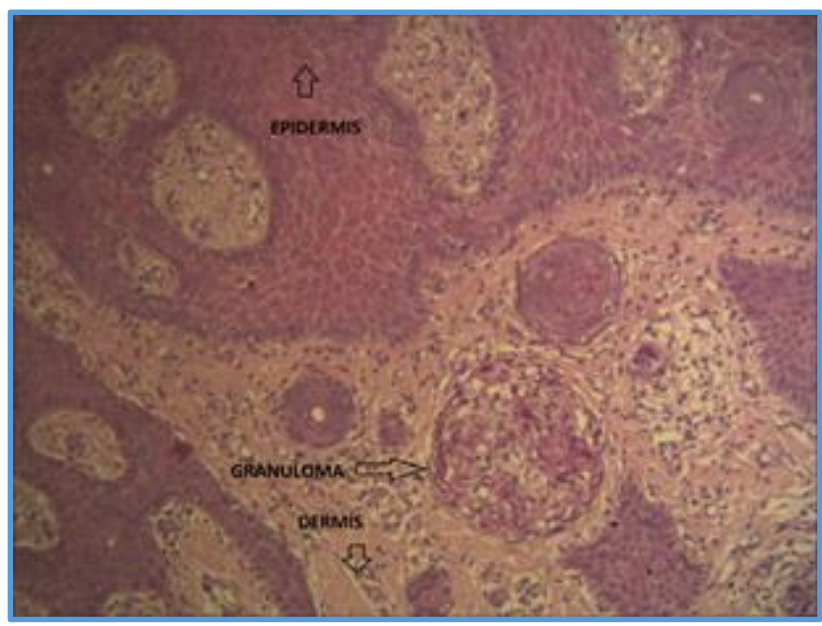

Figure C. H and E: Shows Epidermis, Granuloma and Dermis in a Low Power

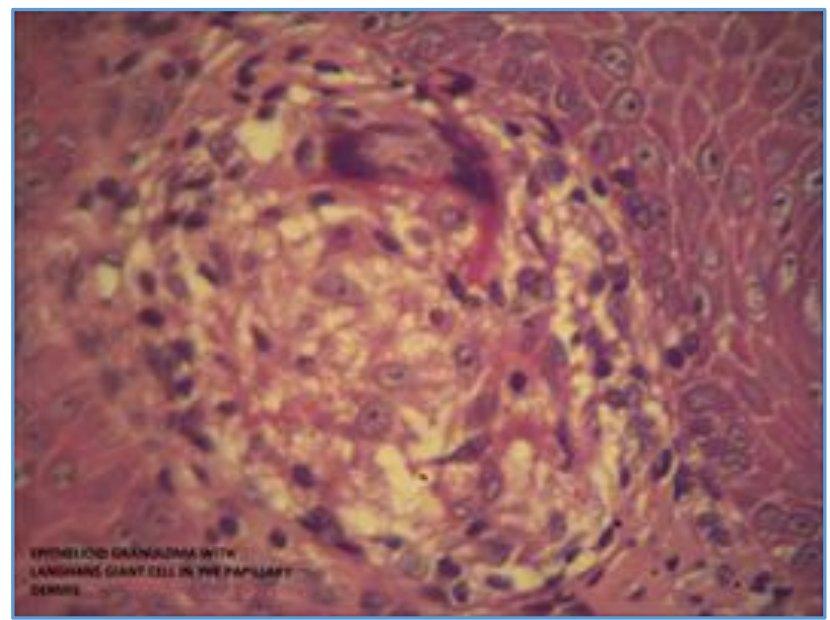

Figure D. Low Power Image showing Epithelioid Granuloma with Giant Cell in the Papillary Dermis

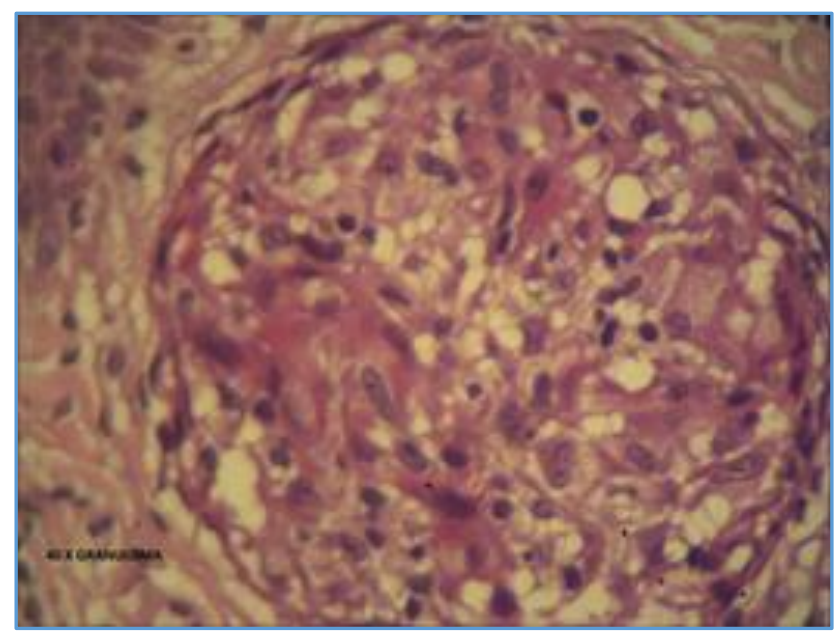

Figure E. 40X Image of the Granuloma

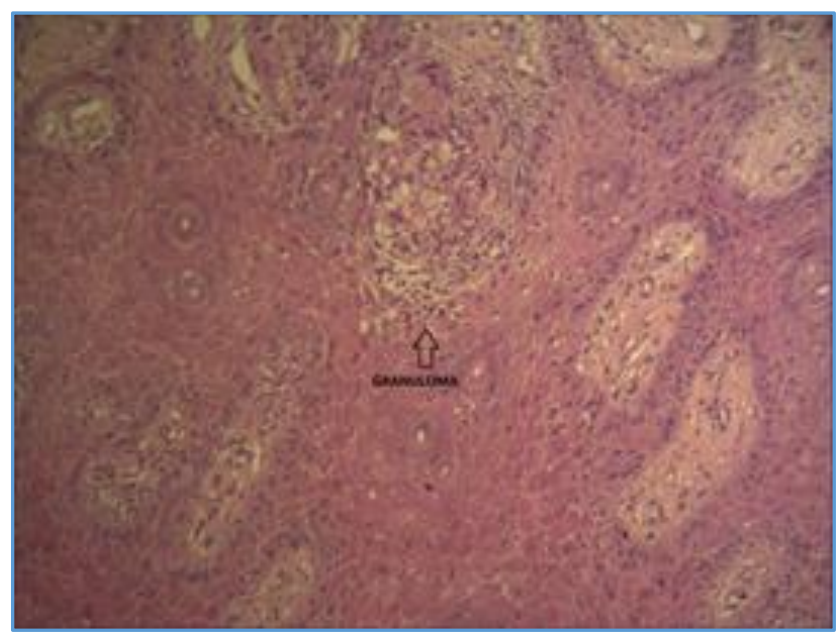

Figure F. 10X Image of the Granuloma

The hallmark of cutaneous TB histology is the presence of characteristic tubercular granulomas with epithelioid cells, Langhans giant cells and lymphocytes. However, characteristics such as the distribution of granulomas in the dermis, nature of cellular infiltrate, presence of necrosis and certain specific epidermal changes aid in classifying and diagnosing the variants of skin TB. 


\section{DISCUSSION OF MANAGEMENT}

In view of the biopsy report, after obtaining Pulmonologist's and Ophthalmologist's opinion the patient was initiated on Category I ATT paediatric kit which consists of Tab. Isoniazid $10 \mathrm{mg} / \mathrm{kg}$, Tab. Rifampicin $15 \mathrm{mg} / \mathrm{kg}$, Tab. Pyrazinamide 35 $\mathrm{mg} / \mathrm{kg}$ and Tab. Ethambutol $20 \mathrm{mg} / \mathrm{kg}$ per day for two months intensive phase and advised to continue Tab. Isoniazid and Tab. Rifampicin for four months as a continuation phase.

Upon followup of the patient after two months the cutaneous lesions showed improvement with no new lesions; hence, the family was advised to continue ATT for the child and review on monthly basis.

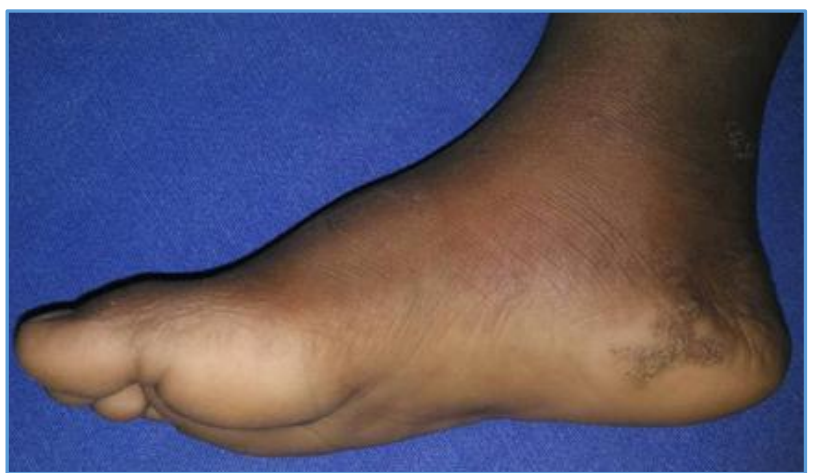

Figure G. Resolution of the Lesion after 2 Months of Followup

Cutaneous TB is treated as per the recommendations of therapy for extrapulmonary TB. Apart from the investigations to establish the diagnosis of cutaneous TB, HIV testing should be carried out in all patients with confirmed or suspected TB, because their HIV status makes a difference to their antitubercular treatment.

As per the latest recommendations (2009) of the World Health Organisation (WHO), cutaneous TB in HIV-negative individuals (adults as well as children) should be treated by directly observed treatment short course (DOTS) chemotherapy consisting of four drugs- isoniazid $(H)$, rifampicin (R), pyrazinamide $(\mathrm{Z})$ and ethambutol (E) given for 2 months (intensive phase) followed by isoniazid and rifampicin given for the next 4 months (continuation phase).[9] Fixed-dose combinations (FDCs) should preferably be used. The drugs may be administered daily or three times weekly. The WHO recommends that daily dosing throughout the duration of therapy ( 2 HRZE/4 HR) is optimal for all newly diagnosed patients with TB. Alternatively, daily intensive phase followed by three times weekly continuation phase [2 HRZE/4 (HR) 3] or three-times weekly dosing throughout therapy [2 (HRZE) 3/4 (HR) 3] may also be used provided that every dose is directly observed. TB patients with known positive HIV status or living in an HIV-prevalent setting should receive daily doses of antitubercular drugs, at least during the intensive phase.[9] For the continuation phase, the optimal dosing frequency for such patients is also daily, although the three-times weekly dosing is an acceptable alternative.

In view of the growing evidence that the use of ethambutol is safe in young children, the WHO recommends that similar to adults this drug should be used in all paediatric cases irrespective of the age.[6] In a case of cutaneous TB where some systemic focus is detected, the corresponding disease categorisation and recommendations should guide the choice of drugs and duration of therapy.

Adverse events caused by anti-TB drugs are much less common in children than in adults. ${ }^{[10]}$ The most important adverse event is the development of hepatotoxicity, which can be caused by isoniazid, rifampicin or pyrazinamide.

\section{FINAL DIAGNOSIS}

Based on the clinical presentation, investigations and histopathological report a final diagnosis of Cutaneous Tuberculosis, Tuberculosis Verrucosa Cutis variant was confirmed and patient responded to the treatment.

Cutaneous tuberculosis (CTB) is the result of a chronic infection by Mycobacterium tuberculosis, M. ovis and occasionally by the Calmette-Guerin bacillus. The clinical manifestations are variable and depend on the interaction of several factors including the site of infection and the host's immunity.[11] The main aetiologic agent of Cutaneous tuberculosis is Mycobacterium tuberculosis (Mtb) that belongs to the class Schizomycetes, order Actinomycetales, family Mycobacteriaceae and genus Mycobacterium. Occasionally, it is also caused by $M$. bovis or BCG vaccine (an attenuated strain of M. bovis).

Mycobacterium tuberculosis is a straight or slightly bent (rod-shaped), non-motile, non-sporulated, unencapsulated bacillus measuring from 1 to $10 \mu \mathrm{m}$ long by 0.2 to $0.6 \mu \mathrm{m}$ wide. Its most important feature is that it becomes stained in red by fuchsin and does not discolour under the actions of alcohol and acid (acid-fast bacillus). Its cellular wall has a high lipid content which grants resistance against the action of chemical agents, though it is susceptible to the action of physical agents (heat and ultraviolet radiation).[12]

Cutaneous tuberculosis can be acquired from haematogenous or lymphatic dissemination of a pulmonary focus or by direct inoculation. The classification of cutaneous TB is shown in Table 1.

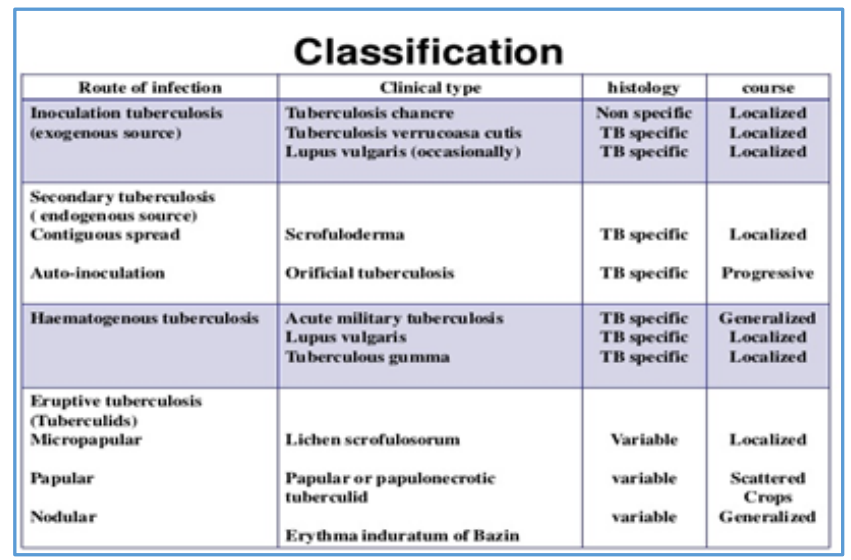

Table 1. Classification and Route of Infection in Cutaneous Tuberculosis

\section{Immunopathogenesis}

The immune response in tuberculosis occurs basically via Th1 pathway with little or no involvement of Th2 pathway. After the mycobacteria are inhaled alveolar macrophages are activated, the infectious agents are internalised and the bactericidal apparatus such as the generation of intermediate nitrogen compounds is triggered in an attempt to eliminate the bacilli at that point.[13,2] 
If the mycobacteria survive, a second stage begins in which they divide within the macrophages. The latter are no longer able to eliminate the infectious agents alone, so they induce the production of cytokines such as IL-6, IL-12, IL-1 $\alpha$ and IL-1 $\beta$, resulting in the recruitment of monocytes, lymphocytes, neutrophils and dendritic cells. CD4+, CD8+ and NK-cell lymphocytes are stimulated by interleukins (IL-12 and IL-18) produced by dendritic cells to release IFN- $\gamma$ in order to stimulate the production of RIN (Reactive Nitrogen Intermediates), ROI (Reactive Oxygen Intermediates) and TNF- $\alpha .[1]$

\section{Tuberculosis Verrucosa Cutis}

This form of the disease, also known as warty TB, occurs due to exogenous inoculation of tubercle bacilli in a previously sensitised individual with good immunity. Clinically, it presents as verrucous papules and plaques with the surface showing fissures or clefts that may extrude pus and often perilesional erythema. It may be difficult to differentiate it from hypertrophic LV as well as verruca vulgaris or common warts.[14]

Tuberculosis verrucosa cutis (TBVC) was once seen in $65.5 \%$ of the childhood cases from Hong Kong, it is an uncommon form of the disease in Indian children. Arya et al have reported TBVC in $15 \%$ of their patients $(3 / 20)$, most of the other series from India have reported it to contribute to $<4.5 \%$ of all cases of paediatric skin TB. Exposed body parts, in particular the lower limbs, are the most common site for warty $\mathrm{TB}$ in children, possibly due to inoculation by unnoticed trauma. In contrast to SFD and LV, lymphadenopathy is not seen.[14,15]

Histology shows hypertrophic changes like pseudoepitheliomatous hyperplasia, the presence of acute infiltrate in the upper dermis and characteristic tuberculoid granulomas in the mid dermis. AFB are rarely demonstrated.

In conclusion, this highlights the importance of keeping TB as an important differential as misdiagnosis or delayed diagnosis of this entity can lead to prolonged morbidity. This case is presented for its rarity of cutaneous tuberculosis in a child below five years of age and that previous vaccination with BCG does not assure a protective role.

\section{REFERENCES}

[1] Pandhi D, Reddy BS, Chowdhary S, et al. Cutaneous tuberculosis in Indian children: the importance of screening for involvement of internal organs. J Eur Acad Dermatol Venereol 2004;18(5):546-51.

[2] Kumar B, Rai R, Kaur I, et al. Childhood cutaneous tuberculosis: a study over 25 years from northern India. Int J Dermatol 2001;40(1):26-32.
[3] Umapathy KC, Begum R, Ravichandran G, et al. Comprehensive findings on clinical, bacteriological, histopathological and therapeutic aspects of cutaneous tuberculosis. Trop Med Int Health 2006;11(10):1521-8.

[4] Sehgal VN, Jain MK, Srivastava G. Changing pattern of cutaneous tuberculosis. A prospective study. Int J Dermatol 1989;28(4):231-6.

[5] Singal A, Pandhi D, Agrawal SK. Multifocal scrofuloderma with disseminated tuberculosis in a severely malnourished child. Pediatr Dermatol 2005;22(5):440-3.

[6] Donald PR, Maher D, Maritz JS, et al. Ethambutol dosage for the treatment of children: literature review and recommendations. Int $\mathrm{J}$ Tuberc Lung Dis 2006;10(12):1318-30.

[7] Ramesh V, Misra RS, Beena KR, et al. A study of cutaneous tuberculosis in children. Pediatr Dermatol 1999;16(4):264-9.

[8] Singal A, Mohanty S, Gandhi V, et al. Cutaneous tuberculosis in paediatric age group. In Proceedings: $7^{\text {th }}$ Congress of European Society for Paediatric Dermatology 2002:33-4.

[9] Treatment of tuberculosis: guidelines. $4^{\text {th }}$ edn. Geneva: World Health Organization, 2009. WHO/HTM/TB/2009.420.

[10] Stop TB Partnership Childhood TB Subgroup, World Health Organization. Guidance for national tuberculosis programmes on the management of tuberculosis in children. Chapter 2: anti-tuberculosis treatment in children. Int J Tuberc Lung Dis 2006;10(11):1205-11.

[11] Global tuberculosis control: epidemiology, strategy, financing: WHO report 2009. Geneva, World Health Organization, 2009 (WHO/HTM/TB/2009.411).

[12] Kaur S, Thami GP, Kanwar AJ, et al. Scrofuloderma with multiple organ involvement in a 5-year-old child. Pediatr Dermatol 2001;18(4):328-31.

[13] Kumar B, Muralidhar S. Cutaneous tuberculosis: a twenty-year prospective study. Int J Tuberc Lung Dis 1999;3(6):494-500.

[14] Wong KO, Lee KP, Chiu SF. Tuberculosis of the skin in Hong Kong. A review of 160 cases. Br J Dermatol 1968;80(7):424-9.

[15] Chong LY, Lo KK. Cutaneous tuberculosis in Hong Kong: a 10-year retrospective study. Int J Dermatol 1995;34(1):26-9. 